\title{
Symmetrization of the Sinc-Galerkin Method for Boundary Value Problems
}

\author{
By John Lund*
}

\begin{abstract}
The Sinc-Galerkin method developed in [5], when applied to the second-order selfadjoint boundary value problem, gives rise to a nonsymmetric coefficient matrix. The technique in [5] is based on weighting the Galerkin inner products in such a way that the method will handle boundary value problems with regular singular points. In particular, the method does an accurate job of handling problems with singular solutions (the first or a higher derivative of the solution is unbounded at one or both of the boundary points). Using $n$ function evaluations, the method of [5] converges at the rate $\exp (-\kappa \sqrt{n})$, where $\kappa$ is independent of $n$. In this paper it is shown that, by changing the weight function used in the Galerkin inner products, the coefficient matrix can be made symmetric. This symmetric method is applicable to a slightly more restrictive set of boundary value problems than the method of [5]. The present method, however, still handles a wide class of singular problems and also has the same $\exp (-\kappa \sqrt{n})$ convergence rate.
\end{abstract}

1. Introduction. The general Galerkin scheme applied to the boundary value problem

$$
\begin{gathered}
L f(x)=f^{\prime \prime}(x)+\mu(x) f^{\prime}(x)+\nu(x) f(x)=\sigma(x), \quad a<x<b, \\
f(a)=f(b)=0,
\end{gathered}
$$

has the discrete form

$$
\left(L f_{m}-\sigma, S_{k}\right)=0, \quad-M \leqslant k \leqslant N,
$$

where the assumed approximate solution of (1.1) is given by

$$
f_{m}(x)=\sum_{p=-M}^{N} f_{p} S_{p}(x) .
$$

The properties of the approximate $f_{m}$ are dependent on both the inner product in (1.2) as well as the choice of the basis functions $S_{k}$ in (1.3). If, for example, the inner product in (1.2) is the $L^{2}(a, b)$ inner product and the $S_{k}$ in (1.3) are $B$-splines, then the above is called the finite element method. If the inner product in (1.2) is a weighted inner product and the $S_{k}$ in (1.3) are sinc functions composed with conformal maps, then the method defined in (1.2) has been termed by F. Stenger the Sinc-Galerkin method. This latter method was developed and (for a specific weight function) thoroughly analyzed in [5]. In that work, the weight function was selected

Received July 15, 1985; revised October 28, 1985.

1980 Mathematics Subject Classification. Primary 65L10, 65N30.

*Work supported by NSF-MONTS Grant \#16404099. 
so that the method would handle boundary value problems (1.1) with regular singular points. In particular, the method works well on (1.1) when the solution $f$ has singularities at $a$ and/or $b$ (a higher derivative of $f$ is unbounded at $a$ and/or $b$ ).

In the selfadjoint case of (1.1):

$$
\begin{gathered}
f^{\prime \prime}(x)+\nu(x) f(x)=\sigma(x), \quad a<x<b, \\
f(a)=f(b)=0,
\end{gathered}
$$

the Sinc-Galerkin coefficient matrix generated by (1.2) is nonsymmetric. This is in contrast to the classical approximation techniques such as finite differences and finite elements as they apply to (1.4). However, as these latter methods are usually based on polynomial approximation, the accuracy of the approximation typically deteriorates in a neighborhood of a singularity of the solution. To compensate for this deterioration, grading the mesh near the singularity (as opposed to a uniform mesh) has been recommended (e.g., [1], [4] or [7]). This mesh grading leads to larger discrete systems in order to maintain the expected asymptotic rate of convergence of the method. This asymptotic rate of convergence depends on the existence and boundedness of higher derivatives of the solution, and as a consequence, standard error methods must be reevaluated when considering singular problems.

Whereas the Sinc-Galerkin method effectively resolves the aforementioned difficulties, it appears to be at the expense of losing the symmetry of the discrete problem. The discrete Galerkin system is very much dependent on the choice of the weight function for the inner product (1.2). The present paper shows in the case of (1.4) that by changing the weight function from what was used in [5], the symmetry of the discrete system is preserved.

In Section 2 the sinc function as well as the class of functions where sinc-function approximation works well is defined. The sinc-quadrature rule, which plays such a fundamental role in the approximation of the inner products for the Sinc-Galerkin method, is briefly summarized. The remainder of the section develops the SincGalerkin method with an arbitrary weight function for the boundary value problem (1.1). This development follows the method in [5] and has been included here for two reasons. Foremost, it seems that the method of [5] leads to the most direct development of the symmetrized Sinc-Galerkin method. Secondly, the method of [5] has been subjected to relatively little numerical testing. Further numerical testing is a natural by-product of the comparison between the method of [5] and the symmetrized Sinc-Galerkin method developed in Section 2.

An error analysis (based on the error formulas of Section 2) is not applicable to the symmetrized Sinc-Galerkin method. However, by changing the dependent variable in the differential equation (1.4), it is shown that the error formulas of Section 2 can be applied to the transformed differential equation. This leads to error formulas for the symmetrized Sinc-Galerkin method. The price one pays for this symmetrization comes in the form of a stronger assumption on the behavior of the true solution $f$ of (1.4) than is required for the weight function used in [5]. The apparent discrepancy between the error formula for the symmetrized Sinc-Galerkin approximate and the nonsymmetric Sinc-Galerkin approximate is also analyzed in Section 2. It is shown that for the appropriate selection of the mesh size, the error for the 
symmetrized Sinc-Galerkin method and the error for the method in [5] are asymptotically equal. The analysis of Section 2 also gives a second choice for the mesh size when using the symmetrized Sinc-Galerkin method. The somewhat surprising numerical results when using this second mesh size are included in the examples of Section 3.

2. Sinc-Galerkin Inner Products. On the whole real line the sinc function is defined by

$$
\operatorname{sinc}(x)=\frac{\sin (\pi x)}{\pi x} .
$$

If $f$ is defined on the real line, then for $h>0$ the series

$$
C(f, h, x)=\sum_{k=-\infty}^{\infty} f(k h) \operatorname{sinc}\left(\frac{x-k h}{h}\right)
$$

is called the Whittaker cardinal expansion of $f$ whenever this series converges. The properties of (2.2), when a finite number of terms is used, has been extensively studied. A comprehensive survey of these approximation properties is found in [6].

For approximations over an arbitrary interval the following definition is needed.

Definition 2.1. Let $D$ be a simply connected domain in the complex $(z=x+i y)$ plane with boundary points $a \neq b$. Let $\phi$ be a conformal map of $D$ onto the strip $S_{d}=\{w: w=u+i v,|v|<d, d>0\}$ such that $\phi(a)=-\infty$ and $\phi(b)=\infty$. If the inverse map of $\phi$ is denoted by $\psi$, define

$$
\Gamma=\{\psi(u):-\infty<u<\infty\}
$$

and

$$
z_{k}=\psi(k h), \quad k=0, \pm 1, \pm 2, \ldots
$$
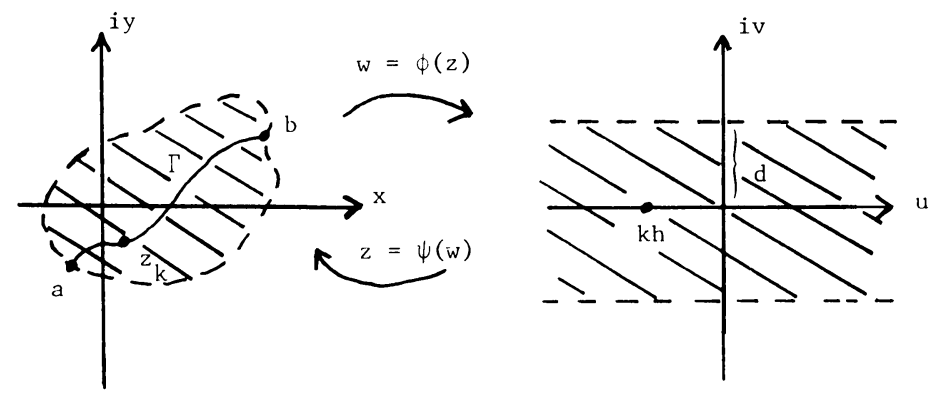

Let $B(D)$ denote the family of functions $F$ which are analytic in $D$ and satisfy

$$
\int_{\psi(u+L)}|F(z) d z| \rightarrow 0, \quad u \rightarrow \pm \infty
$$

where $L=\{i v:|v|<d\}$ and

$$
N(F)=\int_{\partial D}|F(z) d z|<\infty .
$$

The importance of the class $B(D)$ with regard to numerical integration is summarized in the following theorem [6]. 
THEOREM 2.2. If $F \in B(D)$ then

$$
\int_{\Gamma} F(z) d z-h \sum_{p=-\infty}^{\infty} \frac{F\left(z_{p}\right)}{\phi^{\prime}\left(z_{p}\right)}=\frac{i}{2} \int_{\partial D} \frac{F(z) \kappa(\phi, h)(z)}{\sin (\pi \phi(z) / h)} d z
$$

where

$$
\kappa(\phi, h)(z)=\exp \left[\frac{i \pi \phi(z)}{h} \operatorname{sgn}(\operatorname{Im} \phi(z))\right] .
$$

It is (2.6) that provides an accurate approximation of the inner products in the Sinc-Galerkin method.

In this direction, rewrite the orthogonalization of the error for the problem (1.1) in the form

$$
\begin{aligned}
0 & =\int_{\Gamma}(L f-\sigma) S_{k} \circ \phi(z) w(z) d z \\
& =\left(f^{\prime \prime}, S_{k} \circ \phi\right)+\left(\mu f^{\prime}, S_{k} \circ \phi\right)+\left(\nu f, S_{k} \circ \phi\right)-\left(\sigma, S_{k} \circ \phi\right),
\end{aligned}
$$

where $S_{k} \circ \phi(z)=\operatorname{sinc}((\phi(z)-k h) / h)$ and the weight function $w$ for the inner product

$$
(f, g)=\int_{\Gamma} f(z) g(z) w(z) d z
$$

will be specified in what follows. Instead of a direct application of (2.6) to the terms on the right-hand side of (2.8), integration by parts is applied to the inner products to remove the derivatives from $f$. For example, after two integration by parts

$$
\left(f^{\prime \prime}, S_{k} \circ \phi\right)=B_{T_{2}}+\left[f,\left(S_{k} \circ \phi^{\circ} w\right)^{\prime \prime}\right],
$$

where the boundary term is

$$
B_{T_{2}}=\left.f^{\prime} S_{k} \circ \phi w\right|_{a} ^{b}-\left.f\left(S_{k}^{\prime} \circ \phi \phi^{\prime} w+S_{k} \circ \phi w^{\prime}\right)\right|_{a} ^{b},
$$

and $[\cdot, \cdot]$ on the right-hand side of $(2.10)$ is the standard $L^{2}(\Gamma)$-inner product (weight function $w(x) \equiv 1$ in (2.9)). After one integrations by parts, the second term on the right-hand side of (2.8) takes the form

$$
\left(\mu f^{\prime}, S_{k} \circ \phi\right)=B_{T_{1}}+\left[f,\left(\mu S_{k} \circ \phi w\right)^{\prime}\right]
$$

where

$$
B_{T_{1}}=\left.f \mu S_{k} \circ \phi w\right|_{a} ^{b} .
$$

It is to the inner products on the right-hand sides of (2.10) and (2.12) that the quadrature rule (2.6) is applied. For convenience in recording these approximations, the notations

$$
\begin{gathered}
\left.h^{2} \frac{d^{2}}{d \phi^{2}} S_{k} \circ \phi(z)\right|_{z=z_{p}} \equiv \delta_{k p}^{2}= \begin{cases}-\pi^{2} / 3, & p=k, \\
(-1)^{p-k} /(p-k)^{2}, & p \neq k,\end{cases} \\
\left.h \frac{d}{d \phi} S_{k} \circ \phi(z)\right|_{z=z_{p}} \equiv \delta_{k p}^{1}= \begin{cases}0, & p=k, \\
(-1)^{p-k} /(p-k), & p \neq k,\end{cases}
\end{gathered}
$$

and

$$
S_{k} \circ \phi\left(z_{p}\right) \equiv \delta_{k p}^{0}= \begin{cases}1, & p=k \\ 0, & p \neq k\end{cases}
$$


will be useful. Application of (2.6) to (2.10) yields the identity

$$
\left(f^{\prime \prime}, S_{k} \circ \phi\right)=h \sum_{p=-\infty}^{\infty} \frac{f_{p}}{\phi_{p}^{\prime}}\left(S_{k} \circ \phi w\right)_{p}^{\prime \prime}+B_{T_{2}}+E_{F}^{2},
$$

where $g_{p}^{(j)} \equiv g^{(j)}\left(z_{p}\right) \quad(j=0,1,2)$ denotes the $j$ th derivative of a function $g$ evaluated at the point $z_{p}$ in (2.3). The boundary term $B_{T_{2}}$ is given by (2.11) and $E_{F}^{2}$ is the error integral on the right-hand side of (2.6) with $F$ replaced by $f(z)\left(S_{k} \circ \phi w\right)^{\prime \prime}(z)$. Expanding the derivatives under the sum in (2.17) yields

$$
\begin{aligned}
\left(f^{\prime \prime}, S_{k} \circ \phi\right)= & B_{T_{2}}+E_{F}^{2} \\
& +h \sum_{p=-\infty}^{\infty} f_{p}\left[\frac{1}{h^{2}} \delta_{k p}^{2} \phi_{p}^{\prime} w_{p}+\frac{1}{h} \delta_{k p}^{1}\left(\frac{\phi_{p}^{\prime \prime}}{\phi_{p}^{\prime}} w_{p}+2 w_{p}^{\prime}\right)+\delta_{k p}^{0} \frac{w_{p}^{\prime \prime}}{\phi_{p}^{\prime}}\right] .
\end{aligned}
$$

In a similar fashion, (2.12) may be written in the form

$$
\left(\mu f^{\prime}, S_{k} \circ \phi\right)=B_{T_{1}}+E_{F}^{1}-h \sum_{p=-\infty}^{\infty} f_{p}\left[\frac{1}{h} \delta_{k p}^{1}(\mu w)_{p}+\delta_{k p}^{0} \frac{(\mu w)_{p}^{\prime}}{\phi_{p}^{\prime}}\right]
$$

where $B_{T_{1}}$ is defined by (2.13) and $E_{F}^{1}$ is the error integral in (2.6) with $F$ replaced by $f(z)\left(\mu S_{k} \circ \phi w\right)^{\prime}(z)$. To bound the error integrals in (2.18) and (2.19), the inequalities

$$
\begin{gathered}
\left.\frac{1}{2}\left|\frac{S_{k} \circ \phi(z)}{\sin (\pi \phi(z) / h)}\right|\right|_{z \in \partial D} \leqslant C_{0}(h, d) \equiv \frac{h}{2 \pi d} \\
\left.\frac{1}{2}\left|\frac{d\left(S_{k} \circ \phi(z)\right) / d \phi}{\sin (\pi \phi(z) / h)}\right|\right|_{z \in \partial D} \leqslant C_{1}(h, d) \equiv \frac{\pi d+h(\tanh (\pi d / h))}{2 \pi d^{2} \tanh (\pi d / h)},
\end{gathered}
$$

and

$$
\left.\frac{1}{2}\left|\frac{d^{2}\left(S_{k} \circ \phi(z)\right) / d \phi^{2}}{\sin (\pi \phi(z) / h)}\right|\right|_{z \in \partial D} \leqslant C_{2}(h, d) \equiv \frac{C_{1}(h, d)}{d}+\frac{\pi}{2 h d}
$$

will be helpful.

If the weight function $w(x)$ in (2.18) is selected so that $B_{T_{2}}=0$ and $f\left(S_{k} \circ \phi w\right)^{\prime \prime}$ $\in B(D)$, then the computation

$$
\begin{aligned}
& \left|\left(f^{\prime \prime}, S_{k} \circ \phi\right)-h \sum_{p=-\infty}^{\infty} \frac{f_{p}}{\phi_{p}^{\prime}}\left(S_{k} \circ \phi \cdot w\right)_{p}^{\prime \prime}\right|=\left|E_{F}^{2}\right| \\
& \leqslant e^{-\pi d / h} \int_{\partial D}|f(z)| \mid\left\{\left\{S_{k}^{\prime \prime} \phi(z)\left(\phi^{\prime}(z)\right)^{2} w(z)+S_{k} \circ \phi(z) w^{\prime \prime}(z)\right.\right. \\
& \leqslant\left[C_{2}(h, D) N\left(\left(\phi^{\prime}\right)^{2} w f\right)+C_{1}(h, D) N\left(\left(\phi^{\prime \prime} w+2 \phi^{\prime} w^{\prime}\right) f\right)\right. \\
& \left.+C_{0}(h, D) N\left(w^{\prime \prime} f\right)\right] e^{-\pi d / h} \\
& \equiv C_{2} e^{-\pi d / h}
\end{aligned}
$$


follows from the identity

$$
\left.|\kappa(\phi, h)(z)|\right|_{z \in \partial D}=e^{-\pi d / h}
$$

and the estimates (2.5), (2.20)-(2.22). Similarly, if $B_{T_{1}}=0$ in (2.12), then

$$
\begin{aligned}
& \left|\left(\mu f^{\prime}, S_{k} \circ \phi\right)+h \sum_{p=-\infty}^{\infty} f\left(z_{p}\right)\left[\frac{1}{h} \delta_{k p}^{1}(\mu w)_{p}+\delta_{k p}^{0} \frac{(\mu w)_{p}^{\prime}}{\phi_{p}^{\prime}}\right]\right| \\
& \quad \leqslant e^{-\pi d / h}\left[C_{1}(h, D) N\left(\mu w \phi^{\prime} f\right)+C_{0}(h, D) N\left((\mu w)^{\prime} f\right)\right] e^{-\pi d / h} \\
& \quad \equiv C_{1} e^{-\pi d / h} .
\end{aligned}
$$

The two remaining inner products on the right-hand side of (2.8) require no integration by parts. An application of (2.6) to these inner products using (2.16) yields

$$
\left|\left(G, S_{k} \circ \phi\right)-h \frac{G_{k} w_{k}}{\phi_{k}^{\prime}}\right| \leqslant e^{-\pi d / h} C_{0}(h, D) N(G w),
$$

where $G$ is either $\nu f$ or $\sigma$.

For the truncation of the sum in (2.25) ((2.23) is similar), assume that

$$
|f(z) \mu(z) w(z)| \leqslant K \begin{cases}\exp (-\alpha|\phi(z)|), & z \in \Gamma_{a}, \\ \exp (-\beta|\phi(z)|), & z \in \Gamma_{b},\end{cases}
$$

where

$$
\Gamma_{a}=\{\psi(u):-\infty<u \leqslant 0\}, \quad \Gamma_{b}=\{\psi(u): 0<u<\infty\},
$$

and $\psi=\phi^{-1}$. The computation

$$
\sum_{p=N+1}^{\infty}\left|(f \mu w)_{p} \frac{\delta_{k p}^{1}}{h}\right| \leqslant K \sum_{p=N+1}^{\infty} e^{-\beta p h}=\left(\frac{K e^{-\beta(N+1) h}}{1-e^{-\beta h}}\right) \leqslant \frac{K e^{-\beta N h}}{h \beta}
$$

follows upon using (2.15) and $\phi\left(z_{p}\right)=p h$ to obtain the first inequality in (2.29). In a similar fashion,

$$
\sum_{p=-\infty}^{-M-1}\left|(f \mu w)_{p} \frac{\delta_{k p}^{1}}{h}\right| \leqslant \frac{K e^{-\alpha M h}}{\alpha h} .
$$

Using this inequality and (2.29) in (2.25) yields the approximation

$$
\begin{gathered}
\left|\left(\mu f^{\prime}, S_{k} \circ \phi\right)+h \sum_{p=-M}^{N} f\left(z_{p}\right) \frac{\delta_{k p}^{1}}{h}(\mu w)_{p}+h \frac{(\mu w)_{k}^{\prime}}{\phi_{k}^{\prime}} f_{k}\right| \\
\leqslant \frac{K}{\beta} e^{-\beta N h}+\frac{K}{\alpha} e^{-\alpha M h}+C_{1} e^{-\pi d / h},
\end{gathered}
$$

where $C_{1}$ is defined in the last line of (2.25).

For convenience, the preceding inner-product approximations are referenced in a theorem. This is Theorem 2.11 of [5].

THEOREM 2.3. Let $z_{k}$ and $N(\cdot)$ be defined by (2.3) and (2.5), respectively. Let $C_{j}(h, d)$ be given by $(2.20+j), j=0,1,2$. 
(a) If $\sigma$ is analytic in $D$ and $N(\sigma w)<\infty$, then

$$
\left|\left(\sigma, S_{k} \circ \phi\right)-h \frac{\sigma_{k} w_{k}}{\phi_{k}^{\prime}}\right| \leqslant C_{0}(h, D) N(\sigma w) e^{-\pi d / h} .
$$

(b) If $\nu$ and $f$ are analytic in $D$ and $N(\nu f w)<\infty$, then

$$
\left|\left(\nu f, S_{k} \circ \phi\right)-h \frac{\nu_{k} f_{k} w_{k}}{\phi_{k}^{\prime}}\right| \leqslant C_{0}(h, D) N(\nu f w) e^{-\pi d / h} .
$$

(c) If $B_{T_{1}}$ in (2.13) is zero, $f \mu w \phi^{\prime} \in B(D),(\mu w)^{\prime} f \in B(D)$ and (2.27) holds, then (2.30) holds.

(d) Assume $B_{T_{2}}$ in (2.11) is zero, and each of $f w\left(\psi^{\prime}\right)^{2}, f\left[\phi^{\prime \prime} w+\phi^{\prime} w^{\prime}\right]$ and $f w^{\prime \prime}$ are in $B(D)$. Further, assume that $\left|f w \phi^{\prime}\right|$ and $\left|f\left[\phi^{\prime \prime} w+\phi^{\prime} w^{\prime}\right] / \phi^{\prime}\right|$ are bounded along $\Gamma$ by the right-hand side of (2.27). Then

$$
\begin{aligned}
& \left|\left(f^{\prime \prime}, S_{k} \circ \phi\right)-h \sum_{p=-M}^{N} f_{p}\left[\frac{1}{h^{2}} \delta_{k p}^{2} \phi_{p}^{\prime} w_{p}+\frac{\delta_{k p}^{1}}{h}\left(\frac{\phi_{p}^{\prime \prime}}{\phi_{p}^{\prime}} w_{p}+2 w_{p}^{\prime}\right)\right]-h \frac{w_{k}^{\prime \prime}}{\phi_{k}^{\prime}}\right| \\
& \leqslant \frac{K}{\beta h} e^{-\beta N h}+\frac{K}{\alpha h} e^{-\alpha M h}+C_{2} e^{-\pi d / h}
\end{aligned}
$$

where $C_{2}$ is defined in the last line of (2.23).

If the selections

$$
h=(\pi d / \alpha M)^{1 / 2}
$$

and

$$
N=\llbracket \alpha M / \beta \rrbracket
$$

are made for the right-hand sides of (2.30) and (2.33), then the approximations are bounded by $K_{j} M^{(-(j-1) / 2)} e^{-(\pi d \alpha M)^{1 / 2}}(j=1,2)$, respectively. Notice that the selections $h=(\pi d / \beta N)^{1 / 2}$ and $M=\llbracket \beta N / \alpha \rrbracket$ lead to the bounds $K_{j} N^{(-(j-1) / 2)} e^{-(\pi d \beta N)^{1 / 2}}(j=1,2)$ for the right-hand sides of $(2.30)$ and (2.33). The relation between $M$ and $N(=\llbracket \beta N / \alpha \rrbracket$ and $\llbracket \alpha M / \beta \rrbracket)$ shows that the two bounds are asymptotically equal. Here, $K_{j}$ are constants depending on $f, \mu, \nu, w, d, \alpha$, and $\beta$.

The results of Theorem 2.3 are more conveniently recorded with the use of the $m$-vectors $(m=M+N+1)$

$$
\mathbf{S}_{m}(x)=\left(\begin{array}{c}
S_{-M} \circ \phi(x) \\
S_{-M+1} \circ \phi(x) \\
\vdots \\
S_{0} \circ \phi(x) \\
\vdots \\
S_{N} \circ \phi(x)
\end{array}\right), \quad \hat{\mathbf{f}}_{m}=\left(\begin{array}{c}
f\left(z_{-M}\right) \\
f\left(z_{-M+1}\right) \\
\vdots \\
f\left(z_{0}\right) \\
\vdots \\
f\left(z_{N}\right)
\end{array}\right)
$$

and the $m \times m$ matrices

$$
I_{m}^{j}=\left[\delta_{k p}^{j}\right], \quad j=1,2,
$$


where the $k, p$ th entry of $I_{m}^{j}$ is defined by the right-hand side of (2.14) and (2.15), respectively. Let $D_{m}(g)$ be an $m \times m$ diagonal matrix whose diagonal entries are $g\left(z_{-M}\right), g\left(z_{-M+1}\right), \ldots, g\left(z_{0}\right), \ldots, g\left(z_{N}\right)$. Let 1 be an $m$-vector each of whose components are 1 . In this notation the approximate inner products of Theorem 2.3 take the form

$$
\begin{gathered}
\left(\sigma, \mathbf{S}_{m}\right)=h D_{m}\left(\frac{\sigma w}{\phi^{\prime}}\right) \mathbf{1}, \\
\left(\nu f, \mathbf{S}_{m}\right)=h D_{m}\left(\frac{\nu w}{\phi^{\prime}}\right) \hat{\mathbf{f}}_{m}, \\
\left(\mu f^{\prime}, \mathbf{S}_{m}\right)=\left[-I_{m}^{1} D_{m}(\mu w)-h D_{m}\left(\frac{(\mu w)^{\prime}}{\phi^{\prime}}\right)\right] \hat{\mathbf{f}}_{m},
\end{gathered}
$$

and

$$
\left(f^{\prime \prime}, \mathbf{S}_{m}\right)=\left[\frac{1}{h} I_{m}^{2} D_{m}\left(\phi^{\prime} w\right)+I_{m}^{1} D_{m}\left(\frac{\phi^{\prime \prime}}{\phi^{\prime}} w+2 w^{\prime}\right)+h D_{m}\left(\frac{w^{\prime \prime}}{\phi^{\prime}}\right)\right] \hat{\mathbf{f}}_{m}
$$

If the assumed approximate solution of the boundary value problem

$$
\begin{gathered}
f^{\prime \prime}(x)+\mu(x) f^{\prime}(x)+\nu(x) f(x)=\sigma(x), \quad a<x<b, \\
f(a)=f(b)=0,
\end{gathered}
$$

is

$$
f_{m}(x)=\sum_{p=-M}^{N} f_{p} S_{p} \circ \phi(x), \quad m=M+N+1,
$$

then the discrete Sinc-Galerkin system for the determination of the unknown coefficients $\left\{f_{p}\right\}_{-M}^{N}$ is given by

$$
\begin{aligned}
\left\{I_{m}^{2} D_{m}\left(\phi^{\prime} w\right)+h I_{m}^{1} D_{m}\left(\frac{\phi^{\prime \prime} w}{\phi^{\prime}}+2 w^{\prime}-\mu w\right)\right. \\
\left.+h^{2} D_{m}\left(\frac{w^{\prime \prime}-(\mu w)^{\prime}+\nu w}{\phi^{\prime}}\right)\right\} \mathbf{f}_{m}=h^{2} D_{m}\left(\frac{\sigma w}{\phi^{\prime}}\right) \mathbf{1}
\end{aligned}
$$

The matrix equation (2.44) is obtained upon replacing each of the inner products in (2.8) by the approximations in (2.38)-(2.41) and denoting the unknown coefficients in (2.42) by $\mathbf{f}_{m}$.

The case thoroughly analyzed in [5] selects $w(x)=\left[\phi^{\prime}(x)\right]^{-1}$ in (2.44). To see why such a selection is made, consider the problem

$$
\begin{gathered}
f^{\prime \prime}(x)-\frac{1}{x^{2}} f(x)=\frac{1-\log x}{x}, \quad 0<x<1, \\
f(0)=f(1)=0,
\end{gathered}
$$

which has a regular singular point at $x=0$. One of the assumptions in the derivation of the discrete system (2.44) is that the boundary terms $B_{T_{j}}(j=1,2)$ vanish. If the map $\phi_{1}(z)=\log (z /(1-z)$ ) (see Table 3.1) is used in the evaluation of the boundary terms $B_{T_{j}}$, it follows that these terms vanish for $(2.45)(\mu=0$, $\left.\nu=-1 / x^{2}\right)$. Indeed, for any boundary value problem $(2.42)$ on $(0,1)$ each of the boundary terms are zero in the case that (2.42) has regular singular points. The 
problem (2.45) is considered further in Example 3.2. In general, if the selection $w(x)=\left[\phi^{\prime}(x)\right]^{-1}$ is made in (2.44), the error in approximating the true solution of (2.42) by $(2.43)$ is summarized in the following theorem.

THEOREM 2.4. Assume that the coefficients $\mu, \nu$, and $\sigma$ are analytic in the region $D$ of Definition 2.1 and that the problem (2.42) has a unique solution $f$ which is analytic in D. Assume also that

$$
\begin{gathered}
\int_{\partial D}\left|\frac{f^{\prime \prime}(z)}{\phi^{\prime}(z)} d z\right|<\infty, \quad \int_{\partial D}\left|\frac{\mu(z) f^{\prime}(z)}{\phi^{\prime}(z)} d z\right|<\infty \\
\int_{\partial D}\left|\frac{\nu(z) f(z)}{\phi^{\prime}(z)} d z\right|<\infty, \quad \int_{\partial D}\left|\frac{\sigma(z)}{\phi^{\prime}(z)} d z\right|<\infty,
\end{gathered}
$$

and for positive $\alpha$ and $\beta$,

$$
|f(z)| \leqslant K \begin{cases}\exp (-\alpha|\phi(z)|), & z \in \Gamma_{a}, \\ \exp (-\beta|\phi(z)|), & z \in \Gamma_{b},\end{cases}
$$

where $\Gamma_{a}$ and $\Gamma_{b}$ are defined in (2.28). If $h=(\pi d / \alpha M)^{1 / 2}, N=\llbracket \alpha M / \beta \rrbracket$ and the coefficients $\left\{f_{p}\right\}_{-M}^{N}$ in (2.43) are determined from (2.44) with $w(z)=\left[\phi^{\prime}(z)\right]^{-1}$, then

$$
\left|f(z)-f_{m}(z)\right| \leqslant C M^{3 / 2} e^{-(\pi d \alpha M)^{1 / 2}}, \quad z \in \Gamma .
$$

If, however, the selections $h=(\pi d / \beta N)^{1 / 2}$ and $M=\llbracket \beta N / \alpha \rrbracket$ are made, the asymptotically equivalent bound has the form

$$
\left|f(z)-f_{m}(z)\right| \leqslant C N^{3 / 2} e^{-(\pi d \beta N)^{1 / 2}}, \quad z \in \Gamma .
$$

In the special case of (1.4), that is,

$$
\begin{gathered}
f^{\prime \prime}(x)+\nu(x) f(x)=\sigma(x), \quad a<x<b, \\
f(a)=f(b)=0,
\end{gathered}
$$

the discrete Galerkin system (2.44) for the weight $w(x)=\left[\phi^{\prime}(x)\right]^{-1}$ takes the form

$$
\left\{I_{m}^{2}+h I_{m}^{1} D_{m}\left(\frac{-\phi^{\prime \prime}}{\left(\phi^{\prime}\right)^{2}}\right)+h^{2} D_{m}\left(\left(\frac{1}{\phi^{\prime}}\right)\left(\frac{1}{\phi^{\prime}}\right)^{\prime \prime}+\frac{\nu}{\left(\phi^{\prime}\right)^{2}}\right)\right\} \mathbf{f}_{m}=h^{2} D_{m}\left(\frac{\boldsymbol{\sigma}}{\left(\phi^{\prime}\right)^{2}}\right) \mathbf{1}
$$

While (2.50) is a full nonsymmetric matrix system, the rapid rate of convergence guaranteed by Theorem 2.4 often allows one to take a much smaller discrete system than would be the case with, say, finite differences or finite elements. As pointed out in [5], the dominant matrix $I_{m}^{2}$ in the system $(2.50)$ is a symmetric negative-definite matrix with condition number less than $((M+N) / 2+1)^{2}$. Moreover, the approximate solution (2.43) does an accurate job of handling singular solutions of (2.42). Examples 3.1 through 3.5 in the next section bear out this last remark.

If instead of the selection of the weight function $w(x)=\left(\phi^{\prime}(x)\right)^{-1}$, which led to (2.50) for the problem (2.49), one selects $w(x)=\left(\phi^{\prime}(x)\right)^{-1 / 2}$, then $(2.44)$ takes the form

$$
\left\{I_{m}^{2}+h^{2} D_{m}\left(\left(\frac{1}{\left(\phi^{\prime}\right)^{3 / 2}}\right)\left(\frac{1}{\sqrt{\phi^{\prime}}}\right)^{\prime \prime}+\frac{\nu}{\left(\phi^{\prime}\right)^{2}}\right)\right\} \mathbf{y}_{m}=h^{2} D_{m}\left(\frac{\boldsymbol{\sigma}}{\left(\phi^{\prime}\right)^{3 / 2}}\right) \mathbf{1}
$$


where

$$
\mathbf{y}_{m}=D\left(\sqrt{\phi^{\prime}}\right) \mathbf{f}_{m}^{s}
$$

and

$$
f_{m}^{s}(x)=\sum_{p=-M}^{N} f_{p}^{s} S_{p} \circ \phi(x)
$$

is an assumed approximate solution of (2.49). For the remainder of the paper, if the coefficients of $f_{p}$ in (2.43) are determined by (2.50), the method will be called the standard sinc method. If the $f_{p}^{s}$ in (2.53) are determined by (2.51), the method will be referred to as the symmetric sinc method.

The errors in the approximate inner products in Theorem 2.3 remain the same for the present selection of the weight function if

$$
\left|f(z)\left(\phi^{\prime}(z)\right)^{1 / 2}\right| \leqslant K_{s} \begin{cases}\exp \left\{-\alpha_{s}|\phi(z)|\right\}, & z \in \Gamma_{a}, \\ \exp \left\{-\beta_{s}|\phi(z)|\right\}, & z \in \Gamma_{b},\end{cases}
$$

for positive constants $\alpha_{s}$ and $\beta_{s}$, and if on the right-hand side of (2.33) each of $\alpha$ and $\beta$ is replaced by $\alpha_{s}$ and $\beta_{s}$. Moreover, if the selections (2.34) and (2.35) are replaced by

$$
h=\left(\pi d / \alpha_{s} M\right)^{1 / 2}
$$

and

$$
N=\llbracket \alpha_{s} M / \beta_{s} \rrbracket,
$$

then the paragraph following (2.35) reads verbatim in the present case if each $\alpha$ and $\beta$ in that paragraph is replaced by $\alpha_{s}$ and $\beta_{s}$.

The analogue of Theorem 2.4 is unchanged in the present case for functions $f$ satisfying (2.54). However, the assumption on $f$ in (2.54) is more restrictive than is the assumption in (2.46) on $f$. For example, in the case of the map $\phi_{1}(z)=$ $\log (z /(1-z)$ ) (see Table 3.1), the requirement in (2.46) reads (for simplicity, assume that $\alpha=\beta$ )

$$
|f(x)| \leqslant K(x(1-x))^{\alpha}, \quad 0<x<1,
$$

and (2.54) takes the form

$$
|f(x)| \leqslant K_{s}(x(1-x))^{\alpha_{s}+1 / 2}, \quad 0<x<1 .
$$

The assumption on $f$ to obtain (2.50) required that $\alpha>0$. Hence, the assumption in (2.54) restricts the application of the approximation (2.51) to functions $f$ satisfying (2.57) with $\alpha>1 / 2$. Therefore, the approximation defined by the discrete system (2.50) is applicable to a wider class of problems than is the approximation determined by (2.51)

The error in the approximation of the true solution $f$ of (2.49) by (2.53), where the coefficients $\left\{f_{p}^{s}\right\}_{-M}^{N}$ are determined by (2.51) and (2.52), can be related to Theorem 2.4. Make the change of dependent variable

$$
y(x)=\left(\phi^{\prime}(x)\right)^{1 / 2} f(x)
$$


in (2.49) to arrive at the differential equation

$$
y^{\prime \prime}(x)+2 \sqrt{\phi^{\prime}}\left(\frac{1}{\sqrt{\phi^{\prime}}}\right)^{\prime} y^{\prime}(x)+\left(\sqrt{\phi^{\prime}}\left(\frac{1}{\sqrt{\phi^{\prime}}}\right)^{\prime \prime}+\nu\right) y(x)=\sqrt{\phi^{\prime}} \sigma .
$$

Theorem 2.4 applied to the differential equation (2.60) gives the following theorem.

THEOREM 2.5. Assume that $\nu$ and $\sigma$ are analytic in $D$ and that (2.49) has a unique solution $y$ which is analytic in D. Assume also that

$$
\begin{gathered}
\int_{\partial D}\left|\frac{y^{\prime \prime}(z)}{\phi^{\prime}(z)} d z\right|<\infty, \quad \int_{\partial D}\left|\frac{1}{\sqrt{\phi^{\prime}(z)}}\left(\frac{1}{\sqrt{\phi^{\prime}(z)}}\right)^{\prime} y^{\prime}(z) d z\right|<\infty, \\
\int_{\partial D}\left|\frac{\sigma(z)}{\sqrt{\phi^{\prime}(z)}} d z\right|<\infty, \quad \int_{\partial D}\left(\frac{1}{\sqrt{\phi^{\prime}(z)}}\left(\frac{1}{\phi^{\prime}(z)}\right)^{\prime \prime}+\frac{\nu(z)}{\phi^{\prime}(z)}\right) y(z) d z<\infty,
\end{gathered}
$$

and

$$
|y(z)| \leqslant K \begin{cases}\exp \left\{-\alpha_{s}|\phi(z)|\right\}, & z \in \Gamma_{a} \\ \exp \left\{-\beta_{s}|\phi(z)|\right\}, & z \in \Gamma_{b}\end{cases}
$$

If

$$
y_{m}(x)=\sum_{p=-M}^{N} \hat{y}_{p} S_{p} \circ \phi(z)
$$

is an assumed approximate solution of (2.60) and the $\left\{\hat{y}_{p}\right\}_{-M}^{N}$ are determined by (2.51), then with $h=\left(\pi d / \alpha_{s} M\right)^{1 / 2}$ and $N=\llbracket \alpha_{s} M / \beta_{s} \rrbracket$,

$$
\left|y(x)-y_{m}(x)\right| \leqslant C^{s} M^{3 / 2} e^{-\left(\pi d \alpha_{s} M\right)^{1 / 2}} \text {. }
$$

If, however, the selections $h=\left(\pi d / \beta_{s} N\right)^{1 / 2}$ and $M=\llbracket \beta_{s} N / \alpha_{s} \rrbracket$ are made, then the bound in (2.63) takes the form

$$
\left|y(x)-y_{m}(x)\right| \leqslant C^{s} N^{3 / 2} e^{-\left(\pi d \beta_{s} N\right)^{1 / 2}} .
$$

If the inner-product approximations in (2.38) through (2.41) (with $w(z)=$ $\left.\left(\phi^{\prime}(z)\right)^{-1}\right)$ are applied to the differential equation (2.60), then the linear system (2.51) results. Hence, the coefficients $\hat{y}_{p}$ in (2.62) are identical with the coefficients $y_{p}$ $=\sqrt{\phi_{p}^{\prime}} f_{p}^{s}$ in (2.53). That is, substituting (2.52) into (2.53) and using the equality $y_{p}=\hat{y}_{p}$ show that

$$
f_{m}^{s}(x)=\sum_{p=-M}^{N} \frac{y_{p}}{\sqrt{\phi_{p}^{\prime}}} S_{p} \circ \phi(x)=\sum_{p=-M}^{N} \frac{\hat{y}_{p}}{\sqrt{\phi_{p}^{\prime}}} S_{p} \circ \phi(x) .
$$

Recalling that $S_{p} \circ \phi\left(x_{k}\right)=\delta_{k p}^{0}((2.16))$ and using (2.65), (2.59), and (2.63) lead to the estimate

$$
\begin{aligned}
\left|f\left(x_{k}\right)-f_{m}^{s}\left(x_{k}\right)\right| & =\left|\frac{1}{\sqrt{\phi^{\prime}\left(x_{k}\right)}}\right| y\left(x_{k}\right)-y_{k} \mid \\
& =\frac{1}{\left|\sqrt{\phi^{\prime}\left(x_{k}\right)}\right| y\left(x_{k}\right)-y_{m}\left(x_{k}\right) \mid} \\
& \leqslant \frac{C^{s} M^{3 / 2}}{\left|\left(\phi^{\prime}\left(x_{k}\right)\right)^{1 / 2}\right|} e^{-\left(\pi d \alpha_{s} M\right)^{1 / 2}} .
\end{aligned}
$$


Returning to the special case $\phi_{1}(x)=\log (x /(1-x))$ and using

$$
x_{k}=e^{k h} /\left(e^{k h}+1\right)
$$

from Table 3.1, we see that the inequality in (2.66) reads

$$
\left|f\left(x_{k}\right)-f_{m}^{s}\left(x_{k}\right)\right| \leqslant \frac{e^{k h / 2}}{e^{k h}+1} C^{s} M^{3 / 2} e^{-\left(\pi d \alpha_{s} M\right)^{1 / 2}} .
$$

So while the error in the computed $y$ solution in $(2.63)$ is $O\left(e^{-\left(\pi d \alpha_{s} M\right)^{1 / 2}}\right)$, the error in the approximation of the solution $f$ of the original differential equation is, at the nodes, more accurate. To see that this accuracy is asymptotically the same as the error on the right-hand side of (2.67), combine the equality $\alpha=\alpha_{s}+1 / 2((2.57)$ and (2.58)) with the estimate

$$
\frac{1}{2} k h+\left(\pi d \alpha_{s} M\right)^{1 / 2} \simeq(\pi d \alpha M)^{1 / 2}\left(1+\frac{1}{2 \alpha}\left(\frac{k}{M}-\frac{1}{2}\right)\right), \quad \alpha>\frac{1}{2}
$$

in the exponent on the right-hand side of (2.67). The analysis of (2.66) in the case of $(0, \infty)$ boundary value problems for the maps $\phi_{i}(z)(i=2,3)$ in Table 3.1 proceeds in a similar fashion to arrive at the same conclusion: The accuracy of the computed solution $f_{m}^{s}$ (determined by (2.51)) is asympotically the same (at the nodes) as the computed solution $f_{m}$ (determined by (2.50)). In light of these comments, it appears that the selection $h=(\pi d / \alpha M)^{1 / 2}$ (instead of $(2.55)$ ) would lead to errors for the symmetric sinc method (the linear system (2.51)) which are effectively the same as the error for the method of the standard sinc. This is borne out when one surveys the numerical results of the next section. It would seem, based on these numerical comparisons, that the connection between the $f_{p}$ in (2.43) (determined by the nonsymmetric system (2.50)) and the $f_{p}^{s}$ in $(2.53)$ (determined by the symmetric system (2.51)) could be made more explicit than the asymptotic equality implied by (2.67). To analytically establish a result along these lines would require the relationship of the system (2.50) to the system (2.51). At present, this writer does not see any clear relationship between these two systems.

In spite of this last uncertainty, it would seem that the standard sinc method would always be passed over in favor of the symmetric sinc method. In the case of the boundary value problem (2.49), where the true solution satisfies (2.54), this would be the case. If, for example, $(2.49)$ is on $(0,1)$, then the restriction of the symmetric sinc method on the solution requires $\alpha>1 / 2$ in (2.57). The method defined by the discrete system (2.50) requires only that $\alpha>0$. In those examples where $\alpha \in(0,1 / 2]$, the standing of the symmetric sinc method is less than clear. Example 3.3 pursues this point a little further.

It should also be pointed out that the standard sinc method applies to the more general boundary value problem (2.42), whereas the method of this section is limited to the boundary value problem (2.49). If the change of variable $f(x)=$ $u(x) \exp \left(-\frac{1}{2} \int^{x} \mu(t) d t\right)$ is made in order to convert (2.42) to the form

$$
u^{\prime \prime}(x)+g(x) u(x)=\hat{\sigma}(x), \quad u(a)=u(b)=0,
$$

where $g(x)=\mu^{2}(x) / 4+\mu^{\prime}(x) / 2-\nu(x)$ and $\hat{\sigma}(x)=\sigma(x) \exp \left(\frac{1}{2} \int^{x} \mu(t) d t\right)$, then it may be argued that the method of the present section is applicable to (2.68). While this point of view may have potential, it seems that a direct application of the 
standard sinc method to $(2.42)$ is a preferable approach. The main reason for this statement is that the computation of $\hat{\sigma}\left(x_{k}\right)$ in (2.68) would, in general, require the numerical approximation of $m(=M+N+1)$ indefinite integrals in order to define the right-hand side of (2.51).

3. Numerical Implementation. The five examples included in this section were selected in order to illustrate the comparative performance of the standard sinc (2.50) and symmetric sinc (2.51) methods on a set of boundary value problems with various singular behaviors. The formulas required for the assembly of the discrete systems (2.50) and (2.51) are summarized in Table 3.1. The domains $D$ and maps $\phi$ of Definition 2.1 for the special cases when $\Gamma$ is $(0,1)$ and $(0, \infty)$ are illustrated in Figure 3.1. In each of the five examples, the discrete sinc systems defined by $(2.50)$ and (2.51) are used to compute the coefficients $\left\{f_{p}\right\}_{-M}^{N}$ in (2.43) and $\left\{f_{p}^{s}\right\}_{-M}^{N}$ in (2.53), respectively.

\section{TABLE 3.1}

Entries needed for the discrete system (2.50):

$$
\begin{aligned}
& \left\{I_{m}^{2}+h^{2} D_{m}\left(\frac{1}{\left(\phi^{\prime}\right)^{3 / 2}}\left(\frac{1}{\sqrt{\phi^{\prime}}}\right)^{\prime \prime}+\frac{\nu}{\left(\phi^{\prime}\right)^{2}}\right)\right\} D\left(\sqrt{\phi^{\prime}}\right) \mathbf{f}_{m}^{s}=D\left(\frac{\boldsymbol{\sigma}}{\left(\phi^{\prime}\right)^{3 / 2}}\right) \mathbf{1} \\
& \text { and the system (2.51): } \\
& \left\{I_{m}^{2}+h I_{m}^{1} D_{m}\left(\frac{-\phi^{\prime \prime}}{\left(\phi^{\prime}\right)^{2}}\right)+h^{2} D_{m}\left(\frac{1}{\phi^{\prime}}\left(\frac{1}{\phi^{\prime}}\right)^{\prime \prime}+\frac{\nu}{\left(\phi^{\prime}\right)^{2}}\right)\right\} \mathbf{f}_{m}=h^{2} D_{m}\left(\frac{\sigma}{\left(\phi^{\prime}\right)^{2}}\right) \mathbf{1} \text {. } \\
& i \quad \phi_{i}(z) \quad \frac{-\phi^{\prime \prime}}{\left(\phi^{\prime}\right)^{2}} \quad \frac{1}{\phi^{\prime}}\left(\frac{1}{\phi^{\prime}}\right)^{\prime \prime} \quad \frac{1}{\left(\phi^{\prime}\right)^{3 / 2}}\left(\frac{1}{\sqrt{\phi^{\prime}}}\right)^{\prime \prime} \quad \frac{1}{\phi^{\prime}} \quad z_{k} \\
& 1 \log \frac{z}{1-z} 1-2 z \quad 2 z(z-1) \quad-\frac{1}{4} \quad z(1-z) \quad \frac{e^{k h}}{e^{k h}+1} \\
& 2 \begin{array}{llllll}
\log z & 1 & 0 & -\frac{1}{4} & z & e^{k h}
\end{array} \\
& 3 \log (\sinh z) \quad \operatorname{sech}^{2} z \quad \frac{-2 \tanh ^{2}(z)}{\cosh ^{2} z} \quad \frac{3-4 \cosh ^{2} z}{4 \cosh ^{4} z} \quad \tanh z \quad \log \left[e^{k h}+\sqrt{e^{2 k h}+1}\right]
\end{aligned}
$$

For the first three examples (all on $(0,1))$ the condition $(2.46)$ on the solution $f$ of (2.46) is equivalent to

$$
|f(x)| \leqslant K \begin{cases}x^{\alpha}, & 0<x \leqslant 1 / 2 \\ (1-x)^{\beta}, & 1 / 2<x<1 .\end{cases}
$$

All three examples are approximated using the selections (2.34) and (2.35), i.e.,

$$
h=(\pi d / \alpha M)^{1 / 2}, \quad N=\llbracket \alpha M / \beta \rrbracket,
$$

to assemble the discrete systems (2.50) and (2.51). The first and third columns in each of these examples (headed $\Delta f_{k}$ and $\Delta f_{k}^{s}$ under $h=(\pi d / \alpha M)^{1 / 2}$ ) is the absolute error at the nodes $x_{k}=e^{k h} /\left(e^{k h}+1\right),-M \leqslant k \leqslant N$. The second column (headed $\|\Delta f(\alpha)\|)$ is the error between the true solution of (2.42) and the approximate $(2.43)$ on an equispaced grid (mesh size $=.02$ ). The error between the true solution and the approximate defined by (2.53) is effectively the same as the errors listed under $\|\Delta f(\alpha)\|$ and has therefore not been listed. For comparison purposes, the selection $h=\left(\pi d / \alpha_{s} M\right)^{1 / 2}\left(\alpha_{s}=\alpha-1 / 2\right)$ is used in Examples 3.1 and 3.2. 

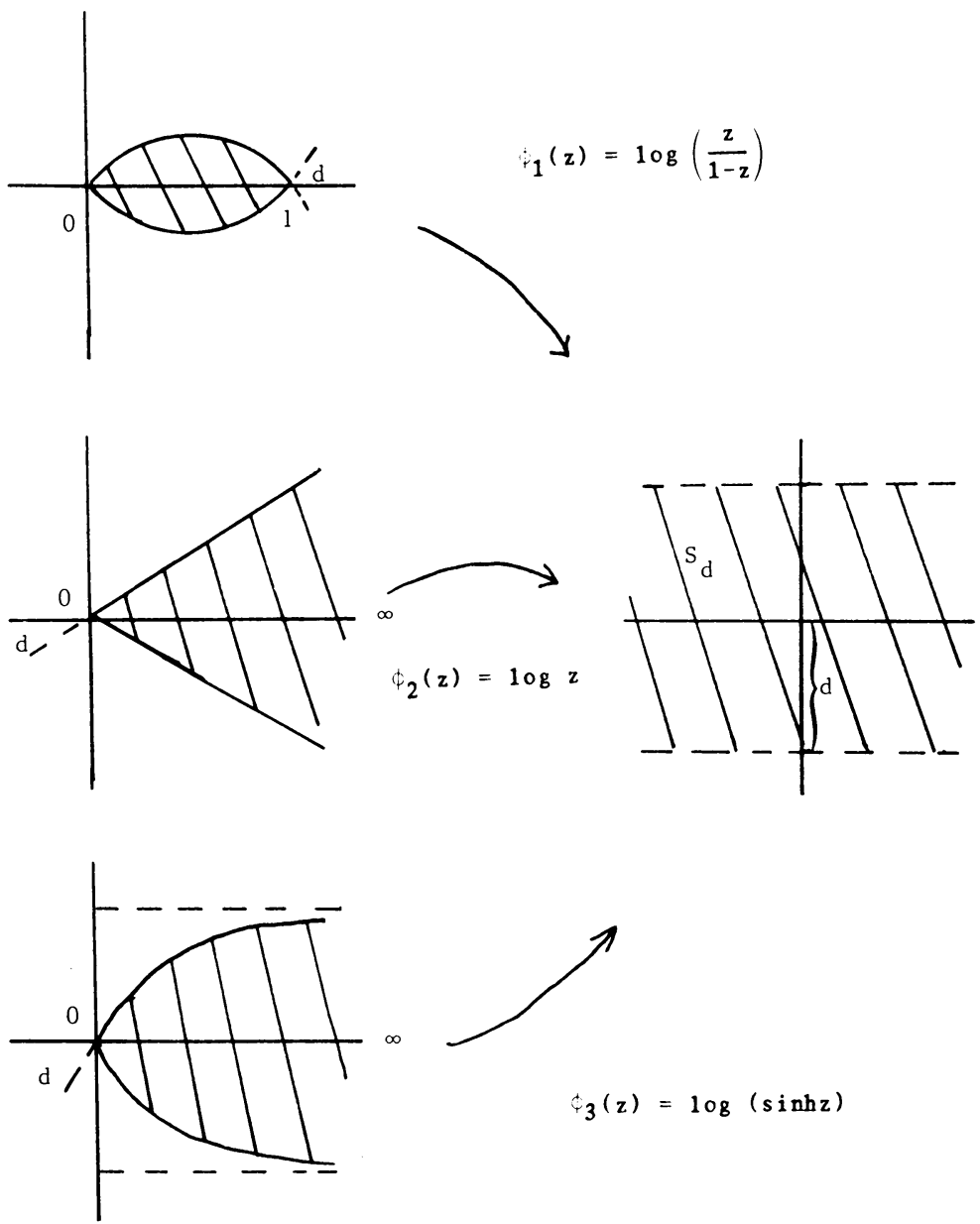

FIGURE 3.1

The domains $D$ and maps $\phi$ of Definition 2.1

The fifth and fourth columns in these two examples (headed $\Delta f_{k}^{s}\left(\alpha_{s}\right)$ and $\left\|\Delta f^{s}\left(\alpha_{s}\right)\right\|$ under $\left.h=\left(\pi d / \alpha_{s} M\right)^{1 / 2}\right)$ are the error at the nodes $e^{k h} /\left(e^{k h}+1\right),-M \leqslant k \leqslant N$, and the uniform error between the true solution of (2.42) and (2.53) on an equispaced grid (mesh size $=.02$ ), respectively. The selection $h=\left(\pi d / \alpha_{s} M\right)^{1 / 2}$ is not available for Example 3.3. This example is, however, approximated using both the discrete systems (2.50) and (2.51). The numerical results support the discussion following (2.67): The error in the approximation of the true solution $f$ by $f_{m}^{s}$ when $h=(\pi d / \alpha M)^{1 / 2}$ is asymptotically the same as the error in approximating $f$ by $f_{m}$ in (2.43).

The choice $h=\left(\pi d / \alpha_{s} M\right)^{1 / 2}$ yields a somewhat unexpected result. A survey of the tables in Examples 3.1 and 3.2 shows not only that the error is within the predicted asymptotic rate (the right-hand side of (2.63)), but that it is somewhat better. Whereas the asymptotic equality in (2.67) indicates that the accuracy for the selection $h=\left(\pi d / \alpha_{s} M\right)^{1 / 2}$ should be about the same as that for the choice 
$h=(\pi d / \alpha M)^{1 / 2}$, there is no reason to expect any increase in accuracy. Based on these numerical computations, one might conjecture that the choice $h=\left(\pi d / \alpha_{s} M\right)^{1 / 2}$ always leads to this increased accuracy. For problems on $(0,1)$, this may well be the case. However, the numerical results of Examples 3.4 and 3.5 indicate that this unexpected accuracy may be map-dependent. Finally, it should be pointed out that in all cases where the selection $h=\left(\pi d / \alpha_{s} M\right)^{1 / 2}$ yielded a gain in accuracy (compared to the choice $\left.h=(\pi d / \alpha M)^{1 / 2}\right)$, the same problem was computed with $h=\left(\pi d / \alpha_{s} M\right)^{1 / 2}$ by the standard sinc method. These results show no increase in accuracy. Based on this discussion the author recommends the selection $h=$ $\left(\pi d / \alpha_{s} M\right)^{1 / 2}$ in all cases when both $\alpha$ and $\alpha_{s}$ are available $(\alpha>1 / 2)$. If, as in Example 3.3, only the selection $\alpha$ is available, the choices provided by (3.2) should be used to define (2.51) for the computation of the approximation defined by (2.53). In all examples one may take $d=\pi / 2$. All examples have zero boundary data and the notation $. x x x-\gamma$ is $. x x x \times 10^{-\gamma}$.

Example 3.1. $f^{\prime \prime}(x)-3 f(x) / 4 x^{2}=-3 \sqrt{x}$. This example was selected since $f(x)$ $=x^{3 / 2}(1-x)$, so that $\alpha=\frac{3}{2} \neq \beta=1$ in (3.1). In this case, $h=\pi / \sqrt{3 M}$ and $N=\llbracket \frac{3}{2} M \rrbracket$. Since $\alpha_{s}=\alpha-\frac{1}{2}=1$, the last two columns of the following table are computed with $h=\pi / \sqrt{2 M}$.

\begin{tabular}{rrccccc}
\multicolumn{9}{c}{$h=(\pi d / \alpha M)^{1 / 2}$} & \multicolumn{2}{c}{$h=\left(\pi d / \alpha_{s} M\right)^{1 / 2}$} \\
$N$ & $M$ & $\Delta f_{k}$ & $\|\Delta f(\alpha)\|$ & $\Delta f_{k}^{s}$ & $\left\|\Delta f^{s}\left(\alpha_{s}\right)\right\|$ & $\Delta f_{k}^{s}\left(\alpha_{s}\right)$ \\
6 & 4 & $.527-2$ & $.638-2$ & $.516-2$ & $.567-2$ & $.786-3$ \\
12 & 8 & $.964-3$ & $.940-3$ & $.947-3$ & $.631-3$ & $.875-4$ \\
15 & 10 & $.926-4$ & $.930-4$ & $.925-4$ & $.252-4$ & $.201-5$ \\
24 & 16 & $.156-4$ & $.157-4$ & $.127-4$ & $.198-5$ & $.119-6$
\end{tabular}

Example 3.2. $f^{\prime \prime}(x)-f(x) / x^{2}=(1-\log (x)) / x$. Here the solution $f(x)=$ $x \log x$ has a logarithmic singularity at $x=0$. The inequality (3.1) is satisfied if $\alpha$ and $\beta$ are in $(0,1)$. The results below are listed for $\alpha=\beta=1$ and $h=\pi / \sqrt{2 M}$. The selection $\alpha_{s}=\frac{1}{2}$ gives $h=\left(\pi d / \alpha_{s} M\right)^{1 / 2}=\pi / \sqrt{M}$.

\begin{tabular}{cccccc} 
& \multicolumn{3}{c}{$h=(\pi d / \alpha M)^{1 / 2}$} & \multicolumn{2}{c}{$h=\left(\pi d / \alpha_{s} M\right)^{1 / 2}$} \\
$M=N$ & $\Delta f_{k}$ & $\|\Delta f(\alpha)\|$ & $\Delta f_{k}^{s}$ & $\left\|\Delta f^{s}\left(\alpha_{s}\right)\right\|$ & $\Delta f_{k}^{s}\left(\alpha_{s}\right)$ \\
4 & $.149-1$ & $.912-2$ & $.148-1$ & $.380-2$ & $.192-2$ \\
8 & $.451-2$ & $.982-3$ & $.454-2$ & $.364-3$ & $.316-3$ \\
16 & $.607-3$ & $.837-4$ & $.609-3$ & $.105-4$ & $.161-4$ \\
24 & $.114-3$ & $.221-4$ & $.114-3$ & $.560-5$ & $.138-5$
\end{tabular}

Example 3.3. $f^{\prime \prime}(x)-(x(1-x))^{-1} f(x)=\sigma(x)$. Here $\sigma(x)$ is selected so that $f(x)=(x(1-x))^{1 / 2}$. Hence, $\alpha=\beta=\frac{1}{2}$ in (3.1), $h=\pi / \sqrt{N}$ and $M=N$ in (3.2). In this case, $\alpha=\frac{1}{2}$ in (2.57), so that $\alpha_{s}=0$ in (2.58). This case is not covered by Theorem 2.4. The approximate solution (2.53) was computed using (2.51) with the parameters $\alpha=\beta=\frac{1}{2}$ and $h=(\pi d / \alpha M)^{1 / 2}$. 


\begin{tabular}{rccl}
\multicolumn{3}{c}{$h=(\pi d / \alpha M)^{1 / 2}$} \\
$M$ & $\Delta f_{k}$ & $\|\Delta f(\alpha)\|$ & $\Delta f_{k}^{s}$ \\
4 & $.245-1$ & $.226-1$ & $.226-1$ \\
8 & $.747-2$ & $.671-2$ & $.741-2$ \\
16 & $.134-2$ & $.123-2$ & $.131-2$ \\
24 & $.346-3$ & $.325-3$ & $.348-3$
\end{tabular}

For the next two examples (both on $(0, \infty)$ ), the condition (2.46) on the solution $f$ of (2.49) is equivalent to

$$
|f(x)| \leqslant K \begin{cases}x^{\alpha}, & x \in(0,1] \\ x^{-\beta}, & x>1\end{cases}
$$

in the case of $\phi_{2}(x)=\log x$, and

$$
|f(x)| \leqslant K \begin{cases}x^{\alpha}, & x \in(0, \log (1+2)] \\ e^{-\beta x}, & x>\log (1+2),\end{cases}
$$

in the case of $\phi_{3}(x)=\log (\sinh (x))$. The selection $N=\llbracket \alpha M / \beta \rrbracket$ in (2.35) again yields the asymptotic convergence rate $\exp \left(-(\pi d \alpha M)^{1 / 2}\right)$. However, in the case of the map $\phi_{2}$, a selection of $N$ leading to a smaller discrete system (2.50) may be made in the case of boundary value problems (2.49) whose solutions have exponential decrease at infinity. To see how this alternative selection is made, assume that the true solution of (2.49) is $O\left(e^{-\gamma x}\right)(\gamma>0, x \rightarrow \infty)$. An inspection of the method leading to the truncation error in (2.33) shows that, at the upper limit,

$$
\left|\sum_{p=N+1}^{\infty}\left[\frac{1}{h} \delta_{k p}^{2}+\delta_{k p}^{1}\left(\frac{-\phi_{p}^{\prime \prime}}{\left(\phi_{p}^{\prime}\right)^{2}}\right)\right] f_{p}\right| \leqslant\left(\frac{\pi^{2}}{3 h}+1\right) \sum_{p=N+1}^{\infty}\left|f\left(x_{p}\right)\right|,
$$

where both (2.14) and (2.15) were used to obtain the inequality in (3.5). The first term to the right-hand side of the inequality in (2.33) is obtained upon replacing $\left|f\left(x_{p}\right)\right|$ in (3.5) by $\exp (-\beta p h)$ (from (3.3)) and summing the resulting series. In the , present setting, $f\left(x_{p}\right) \simeq \exp \left(-\gamma e^{p h}\right)$, so that the truncation error is much smaller than is indicated by the general development leading to (2.33). Regarding the truncation error in (3.5) as $\exp \left(-\gamma e^{N h}\right)$, and equating this to $\exp (-\alpha M h)$, leads to the selection

$$
N=\llbracket \frac{1}{h} \log \left(\frac{\alpha}{\gamma} M h\right)+1 \rrbracket .
$$

This procedure, $h=(\pi d / \alpha M)^{1 / 2}$ and $N$ selected as in (3.6), is elaborated on in Example 3.4.

As is the case for the finite-interval example, there is also the choice $h=$ $\left(\pi d / \alpha_{s} M\right)^{1 / 2}$. In contrast, however, to the finite-interval examples, the error in the computed solution $f_{m}^{s}$ using $h=\left(\pi d / \alpha_{s} M\right)^{1 / 2}$ displays no increased accuracy as compared to the error in the computed solution $f_{m}^{s}$ when $h=(\pi d / \alpha M)^{1 / 2}$ and the 
map $\phi_{2}$ is used. Example 3.5 indicates that the unexpected increase in accuracy seen in the finite-interval examples may persist for the map $\phi_{3}$. In the tables that follow, the columns headed $\Delta f_{\phi_{2}}$ and $\Delta f_{\phi_{2}}^{s}$ denote the error at the nodes $x_{k}=e^{k h}$ $(-M \leqslant k \leqslant N)$ between the true solution of (2.49) and its approximate $f_{m}$ (determined by (2.50)) and $f_{m}^{s}$ (determined by (2.51)). The columns headed $\Delta f_{\phi_{3}}$ and $\Delta f_{\phi_{3}}^{s}$ denote this error for the map $\phi_{3}(x)$.

Example 3.4. $f^{\prime \prime}(x)-f(x)=-2 e^{-x}$. The solution $f(x)=x e^{-x}$ satisfies (3.3) and (3.4) for $\alpha=\beta=1$. Hence, if we use (3.2), the selections $h=(\pi d / \alpha M)^{1 / 2}=$ $\pi / \sqrt{2 M}$ and $M=N$ are applicable for either of the maps $\phi_{2}$ or $\phi_{3}$. However, due to the exponential decrease of the solution, $N$ is selected for the map $\phi_{2}$ by (3.6) $(\gamma=1)$. Note the increased accuracy for the choice $h=\left(\pi d / \alpha_{s} M\right)^{1 / 2}$ in the case of the map $\phi_{3}(x)$.

\begin{tabular}{|c|c|c|c|c|}
\hline \multirow[b]{2}{*}{$M$} & \multirow[b]{2}{*}{$N$} & \multicolumn{2}{|c|}{$h=(\pi d / \alpha M)^{1 / 2}$} & \multirow{2}{*}{$\begin{array}{c}h=\left(\pi d / \alpha_{s} M\right)^{1 / 2} \\
\Delta f_{\phi_{2}}^{s}\left(\alpha_{s}\right)\end{array}$} \\
\hline & & $\Delta f_{\phi_{2}}$ & $\Delta f_{\phi_{2}}^{s}$ & \\
\hline 4 & 2 & $.631-2$ & $.551-2$ & $.283-1$ \\
\hline 8 & 4 & $.124-2$ & $.949-3$ & $.534-2$ \\
\hline 16 & 6 & $.844-4$ & $.857-4$ & $.580-3$ \\
\hline \multirow[t]{2}{*}{24} & 7 & $.169-4$ & $.127-4$ & $.174-3$ \\
\hline & & \multicolumn{2}{|c|}{$h=(\pi d / \alpha M)^{1 / 2}$} & $h=\left(\pi d / \alpha_{s} M\right)^{1 / 2}$ \\
\hline \multicolumn{2}{|c|}{$M=N$} & $\Delta f_{\phi_{3}}$ & $\Delta f_{\phi_{3}}^{s}$ & $\Delta f_{\phi_{3}}^{s}\left(\alpha_{s}\right)$ \\
\hline \multicolumn{2}{|c|}{4} & $.638-2$ & $.564-2$ & $.212-2$ \\
\hline \multicolumn{2}{|c|}{8} & $.195-2$ & $.188-2$ & $.363-3$ \\
\hline \multicolumn{2}{|c|}{16} & $.271-3$ & $.269-3$ & $.198-4$ \\
\hline \multicolumn{2}{|c|}{24} & $.591-4$ & $.516-4$ & $.605-5$ \\
\hline
\end{tabular}

Example 3.5. $f^{\prime \prime}(x)-2 x f(x) /\left(x^{2}+1\right)^{2}=-6 x /\left(x^{2}+1\right)^{3}$. The solution $f(x)=$ $x /\left(x^{2}+1\right)$ satisfies the assumption (3.3), but not (3.4). Hence, the rate of convergence given by Theorem 2.4 is obtained using the map $\phi_{2}(z)=\log z$ with $\alpha=\beta=1$, $h=\pi / \sqrt{2 M}$ and $M=N$. The map $\phi_{3}$ yields a convergent method for this example, but the rate of convergence is very slow. Problems where the map $\phi_{3}$ is preferable to the map $\phi_{2}$ are discussed in [2], [3] and [6, p. 210]. Note, just as in the last example, the error for the selection $h=\left(\pi d / \alpha_{s} M\right)^{1 / 2}$, while within the asymptotic rate given by $\exp \left(-\left(\pi d \alpha_{s} M\right)^{1 / 2}\right)$, is not as good as the error using the selection $h=$ $(\pi d / \alpha M)^{1 / 2}$.

\begin{tabular}{rccc} 
& \multicolumn{2}{c}{$h=(\pi d / \alpha M)^{1 / 2}$} & $h=\left(\pi d / \alpha_{s} M\right)^{1 / 2}$ \\
$M$ & $\Delta f_{\phi_{2}}$ & $\Delta f_{\phi_{2}}^{s}$ & $\Delta f_{\phi_{2}}^{s}\left(\alpha_{s}\right)$ \\
4 & $.126-1$ & $.121-1$ & $.586-1$ \\
8 & $.105-2$ & $.159-2$ & $.130-1$ \\
16 & $.118-3$ & $.107-3$ & $.123-2$ \\
24 & $.229-4$ & $.452-4$ & $.234-3$
\end{tabular}


Acknowledgment. The author thankfully acknowledges the revisions suggested by the two referees which have greatly improved the presentation of this article. The author is also grateful for the excellent typing of Mrs. Susie Gray.

Department of Mathematical Sciences

Montana State University

Bozeman, Montana 59717

1. L. Kaufman \& D. D. Warner, "High-order, fast-direct methods for separable elliptic equations," SIAM J. Numer. Anal., v. 21, 1984, pp. 672-694.

2. J. LUND, "Sinc function quadrature rules for the Fourier integral," Math. Comp., v. 41, 1983, pp. 103-113.

3. J. LUND \& B. V. RiLEY, "A Sinc-collocation method for the computation of the eigenvalues of the radial Schrödinger equation," IMA J. Numer. Anal., v. 4, 1984, pp. 83-98.

4. S. W. Schоombie \& J. F. Botha, "Error estimates for the solution of the radial Schrödinger equation by the Rayleigh-Ritz finite element method," IMA J. Numer. Anal., v. 1, 1981, pp. 47-63.

5. F. STENGER, "A Sinc-Galerkin method of solution of boundary value problems," Math. Comp., v. 33, 1979, pp. 85-109.

6. F. STENGER, "Numerical methods based on Whittaker cardinal, or sinc functions," SIAM Rev., v. 23, 1981, pp. $165-224$.

7. A. Weiser, S. C. Eisenstat \& M. M. Schultz, "On solving elliptic problems to moderate accuracy," SIAM J. Numer. Anal., v. 17, 1980, pp. 908-929. 